\title{
Aplicabilidad del modelo ASSURE en la didáctica de lenguas
}

Recibido: 05/10/2015

Aceptado: 08/03/2016

\author{
Applicability of ASSURE model in TESOL
}

Hilda Buitrago, hildacbg@gmail.com

Universidad Cooperativa de Colombia

\section{Resumen}

El presente trabajo examina los lineamientos del Ministerio de Educación Nacional respecto a la enseñanza de una segunda lengua, sus objetivos, alcance y requerimientos didácticos y tecnológicos para alcanzar las metas de aprendizaje propuestas. Posteriormente, describe los pasos del modelo de diseño instruccional ASSURE y explica las razones por las cuales este tipo de diseño permite la puesta en práctica de algunos de los principales postulados teóricos en los que se basa la didáctica de los idiomas extranjeros y el uso efectivo de las TIC para fomentar la participación del estudiante en los procesos de enseñanza-aprendizaje. Se recomiendan estudios adicionales que permitan determinar con mayor precisión la eficacia del Modelo Instruccional ASSURE en las modalidades e-learning y blended learning.

Palabras claves: Educación virtual, diseño instruccional, TIC, didáctica de lenguas.

\section{Abstract}

This paper examines the standards issued by the Colombian Ministry of Education regarding second language teaching, its objectives, scope and teaching, and technological requirements in order to achieve learning goals. It later describes the steps of the ASSURE instructional design model and explains the reasons why this type of design allows for the implementation of some of the main theoretical postulates about foreign language teaching and effective ICT use that encourage student participation in the intended teaching-learning processes. Further research is recommended to more accurately measure the effectiveness of the ASSURE instructional model in the e-learning and blended learning modalities for teaching a second language, in order to determine the extent to which the face-to-face sessions are necessary and how they can be enriched through the use of ICT.

Keywords: E-learning, instructional design, ICT, language teaching. 


\section{Introducción}

Hoy en día, las políticas que rigen la enseñanza de una segunda lengua en casi cualquier lugar del mundo conllevan la provisión de oportunidades de contacto y experiencia con la lengua objeto de estudio y las culturas en las que esta se usa. Colombia no es la excepción y el Ministerio de Educación Nacional (MEN) ha diseñado políticas claras que buscan proporcionar a los estudiantes los conocimientos lingüísticos necesarios para ser competitivos a nivel académico y laboral en la sociedad globalizada donde deberán desempeñarse. Esto sólo se puede lograr a través de iniciativas pedagógicas que promuevan el trabajo participativo y colaborativo a través de diseños tecno-pedagógicos que tengan en cuenta las necesidades y expectativas de los educandos. El dominio de una segunda lengua y debida apropiación de las posibilidades académicas y laborales que ofrecen las TIC convierten la enseñanza de una segunda lengua en un desafío que ninguna sociedad debe desatender.

Este documento presenta diversas consideraciones teóricas relacionadas con la enseñanza de una segunda lengua, el papel de las TIC en la educación y el diseño instruccional. Se da especial relevancia al modelo instruccional ASSURE, ya que este conjuga varios de los principios teóricos antes mencionados y permite su aplicación eficiente en situaciones de aprendizaje con herramientas tecnológicas, ya sea en modalidad e-learning o blended learning. La finalidad del artículo es ofrecer una panorámica de la didáctica de lenguas extranjeras en Colombia apoyada por la tecnología, su interrelación con el diseño tecno-pedagógico y la idoneidad del modelo ASSURE para diseñar diversos tipos de actividades pedagógicas de corta o larga duración que promuevan un aprendizaje constructivista e interconectado de una segunda lengua, tal como lo propone el Ministerio de Educación Nacional.

\section{1 ¿Para qué aprender un segundo idioma?}

Nunca antes en la historia de la humanidad ha sido tan importante aprender una segunda lengua como ahora. Diversos factores como la globalización de la economía, la comunicación y la educación, entre otros, hacen necesario formar individuos con competencias lingüísticas en al menos un idioma diferente a la lengua materna. Esto trae no pocos retos a la educación en general y a los docentes en particular. Si a todo esto se le agrega la presencia inevitable de la tecnología de la comunicación, se evidencia un reto y también una oportunidad valiosa para que el diseño instruccional ofrezca las soluciones que la educación, tanto presencial como virtual, requiere. El ritmo tecnológico al que se ve expuesta la sociedad actual hace apremiante este tipo de intervención por parte de los diseñadores instruccionales con el objetivo de proporcionar a los estudiantes de una segunda lengua las acciones formativas idóneas para alcanzar los objetivos educativos y las competencias lingüísticas, sociales y culturales propuestas. Con el fin de ofrecer a los estudiantes un aprendizaje que les permita desenvolverse competitivamente en el ámbito internacional sin que se vea afectado su sentido de pertenencia a una cultura, se han ido desarrollando diversos modelos de diseño instruccional como el ASSURE. Este modelo en particular ofrece a los docentes de lenguas extranjeras las posibilidades y el desafío de explorar una amplia gama de recursos tecnológicos y actividades pedagógicas conducentes al cumplimiento de los lineamientos del Ministerio de Educación Nacional, respecto a lo que la enseñanza efectiva de una segunda lengua debe contemplar.

\section{Objetivos de la enseñanza de una segunda lengua}

Toda acción educativa debe ser planificada teniendo en cuenta los objetivos que se pretenden alcanzar. Una de las razones más importantes para enseñar y aprender una segunda lengua es que esta permite al estudiante tener una visión más crítica, flexible, metalingüística e interactiva de su propio idioma 
y de su cultura (Altablero, 2005). El aprendizaje de otro idioma posibilita además el desarrollo de competencias sociolingüísticas e interculturales necesarias para comunicarse efectivamente en diversos entornos y circunstancias. Esto requiere el diseño de programas educativos que involucren estrategias integradoras, uso efectivo de las TIC y adecuados medios de evaluación (MEN, s.f.).

Es importante señalar que los objetivos de la enseñanza de idiomas han ido evolucionando a la par con las diversas teorías del aprendizaje y con los requerimientos de la sociedad actual. En consecuencia, se observa una evolución de la competencia meramente lingüística a la intercultural. Los objetivos de la enseñanza de una segunda lengua deben tener en cuenta tres niveles de concreción que son: la proficiencia, la competencia y la función. Estos niveles llevan al aprendiz a usar la lengua no solamente con fluidez sino también efectivamente en situaciones y con interlocutores específicos para cumplir funciones comunicativas concretas (Ministerio de Educación de Cultura y Deporte de España - MECD, 2002). Lo anterior ofrece una visión del lenguaje como un instrumento de comunicación social y no sólo como un objeto de estudio que da lugar a los enfoques comunicativos propios de la enseñanza de un idioma extranjero (Trujillo, 2001).

Teniendo en cuenta lo anterior, es posible afirmar que el aprendizaje de una segunda lengua requiere el desarrollo de competencias comunicativas y tecnológicas específicas que posibiliten los niveles de concreción descritos. Indudablemente, uno de los ámbitos más importantes a tener en cuenta en cualquier propuesta de enseñanza de una segunda lengua es el comunicativo (MEN,s.f.). Es precisamente la dimensión comunicativa la que permitirá que los estudiantes se familiaricen con la segunda lengua y con otras culturas al interactuar con individuos y organizaciones para expresar sus propias ideas y reflexionar a partir de la información y percepción que reciban. Esto hace indispensable que se diseñen acciones formativas que ofrezcan oportunidades de hablar y escribir en la segunda lengua en contextos reales, ya que es este tipo de lenguaje el que fundamenta el desarrollo de las 4 habilidades lingüísticas básicas: comprensión lectora, comprensión auditiva, capacidad para hablar y escribir. Un diseño instruccional que proporcione actividades que permitan a los estudiantes comunicarse en forma efectiva, creativa y ética en la segunda lengua tendrá mayores posibilidades de éxito.

Otros dos ámbitos que no se deben perder de vista son los de la cultura y las conexiones, ya que los estudiantes requieren desarrollar una visión más amplia de otros tipos de manifestaciones culturales, patrones de comportamiento, perspectivas y estilos de vida. Esto es posible a través de las conexiones y el intercambio de información e ideas que realice (MECD, 2002). No basta con diseñar actividades que permitan compilar información sobre las otras culturas; es crucial que a los estudiantes se les permita involucrarse en interacciones directas y significativas con individuos de esa cultura. Un diseño instruccional exitoso requiere, por tanto, planear actividades que lleven a los estudiantes más allá de las experiencias de aprendizaje formal y les permitan desarrollar una mayor sensibilidad frente al saber y al hacer. 


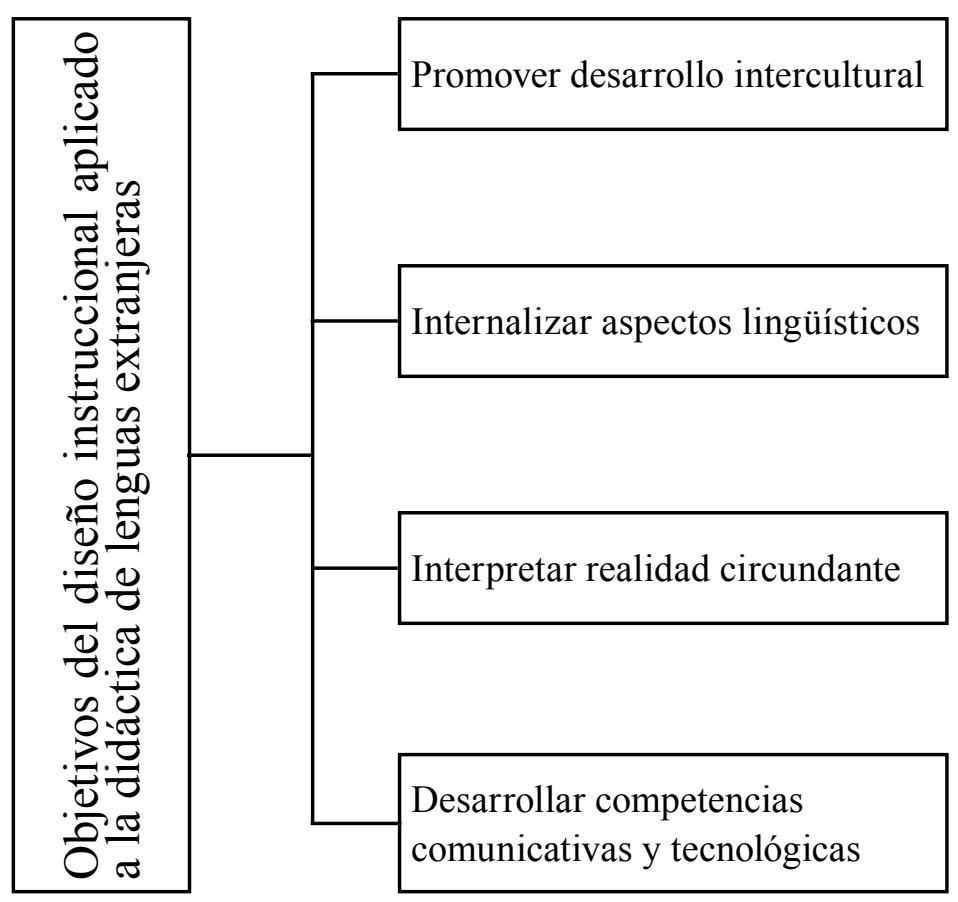

Figura 1. Objetivos del diseño instruccional en didáctica de lenguas

Como se observa en la figura 1, uno de los objetivos más importantes de un programa de enseñanza de segunda lengua debe ser entonces el involucrar al estudiante en procesos que lo lleven a un desarrollo intercultural, entendiéndose éste como el intercambio respetuoso y reflexivo de conocimientos y creencias que se va llevando a cabo a medida que se internalizan las particularidades lingüísticas del idioma que se está aprendiendo. En otras palabras, como lo propone el Ministerio de Educación Nacional (s.f.), deben diseñarse programas que permitan a los estudiantes el uso del nuevo idioma para aprender e interpretar la realidad y para comunicar ideas, sentimientos y opiniones en una variedad de situaciones y contextos.

\section{El Aprendizaje de una segunda lengua}

Se considera el aprendizaje como un proceso de cambio conductual, lo cual implica la «adquisición y modificación de conocimientos, estrategias, habilidades, creencias y actitudes» (Ecured, 2017). Teniendo en cuenta esto, es fundamental diseñar acciones pedagógicas que permitan este tipo de cambio y en el desarrollo de capacidades cognitivas, afectivas y sociales específicas. Basados en los trabajos de Bruner, Vygotsky, Piaget, Seaver y Botel, Goodman et al. (1987; citado en MEN, s.f., p. 13-14), proponen cuatro principios básicos del aprendizaje. Estos principios privilegian el significado, el contexto social, las relaciones entre los diversos procesos lingüísticos y el papel del ser humano. Estos autores coinciden en afirmar que el aprendizaje requiere la participación activa, colaborativa y reflexiva a través de cinco tipos de experiencias que deben ser incluidos en cualquier metodología usada para enseñar una segunda lengua. Estas cinco experiencias son: «leer textos, componer textos de varios géneros, usar la lectura y la escritura para adquirir nuevos conocimientos fuera de la escuela, analizar las funciones lingüísticas y aprender a aprender» (MEN, 2006, p. 14). Para Krashen (1982, citado en MEN, s.f., p. 14) el papel protagónico que tiene el significado en el proceso de aprendizaje de una lengua extranjera hace indispensable que los actos comunicativos sean 
comprensibles para el estudiante. Por otra parte, Frank Smith (1989, citado en MEN, 2006, p. 14), afirma que «un lenguaje comprensible debe ser real, natural, integrado, contextualizado, interesante, relevante para el estudiante, relacionado con eventos reales, socialmente útil». Añade además que el estudiante debe apropiarse de dicho lenguaje y elegir cómo usarlo.

\section{$4 \quad$ Principios básicos de la enseñanza de una segunda lengua}

En resumen, según Brown (1995, citado en MEN, s.f., p. 14), existen cinco principios que deben tenerse en cuenta en la enseñanza-aprendizaje de un segundo idioma. Estos principios son:

1. Reconocer y aprovechar la motivación del estudiante

2. Dar oportunidades al estudiante de arriesgarse en el manejo de la lengua extranjera, tanto en lo oral como en lo escrito

3. Reconocer que el lenguaje y la cultura están estrechamente relacionados

4. Fomentar la auto-confianza del estudiante

5. Ser realistas en cuanto al tiempo que se necesita para aprender una lengua extranjera

\section{Recomendaciones metodológicas para la enseñanza de lenguas extranjeras}

Las metodologías para enseñar lenguas extranjeras deben apuntar a la optimización de los procesos de aprendizaje. En el campo de las lenguas extranjeras, existen ciertos requerimientos metodológicos, sintetizados en la figura 2, para garantizar una mayor eficiencia en los procesos de enseñanzaaprendizaje (MEN, s.f.). Estas metodologías deben caracterizarse por ser activas, interactivas y lúdicas, permitiendo así que el estudiante aprenda haciendo y participando. Esto lo convierte en un agente activo de su propio proceso de aprendizaje de la lengua, capaz de construir conocimiento y conexiones con otros individuos a través de actividades que fomenten la socialización. Adicionalmente, la enseñanza de una lengua extranjera requiere de intervenciones pedagógicas significativas e interesantes centradas en el estudiante y que reflejen sus intereses y necesidades. Estas acciones formativas deben potenciar su desarrollo y facilitar su aprendizaje por medio del descubrimiento de sus propios estilos y estrategias para aprender. Lo anterior es posible si se integra el conocimiento previo con el nuevo, con el fin de promover reflexiones metacognitivas sobre el aprendizaje de la segunda lengua y si se implementan acciones metodológicas flexibles e innovadoras que ofrezcan una variedad de actividades, técnicas y procedimientos que involucren componentes afectivos y culturales que faciliten el acercamiento entre individuos y culturas. 


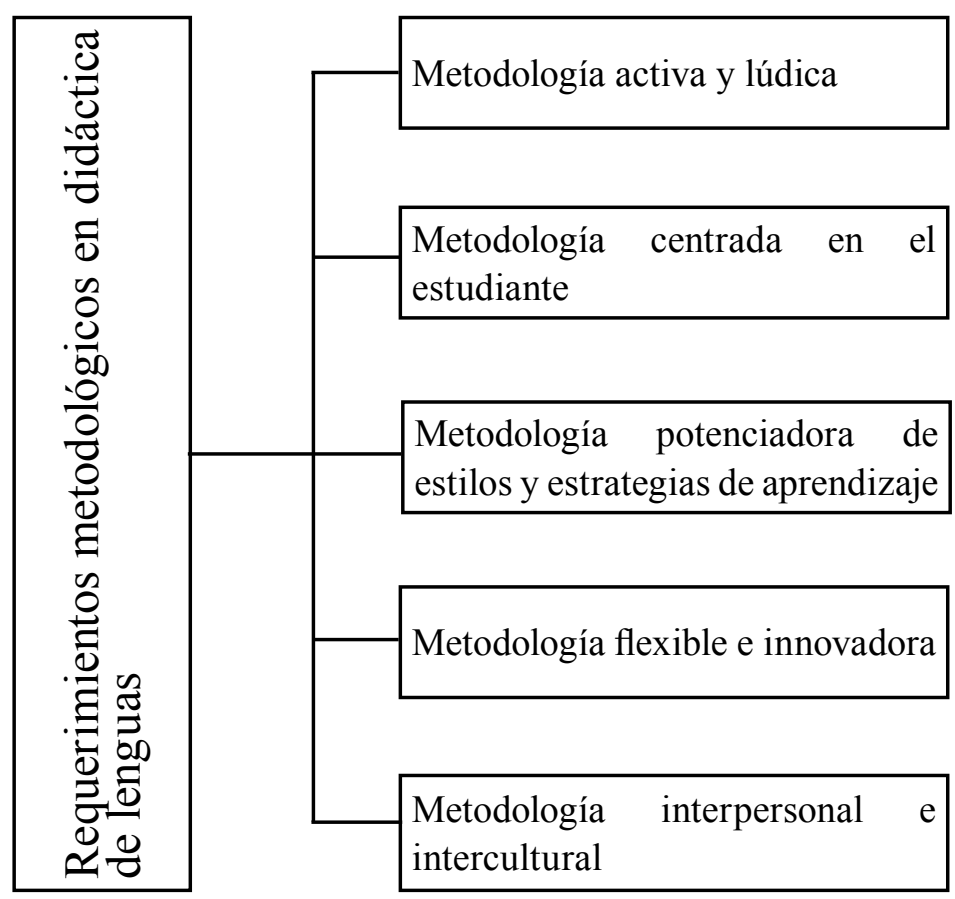

Figura 2. Requerimientos metodológicos en didáctica de lenguas

\section{$6 \quad$ El uso de las TIC en la enseñanza de lenguas extranjera}

Los avances tecnológicos actuales permiten un mejoramiento continuo y un mayor alcance de los procesos de enseñanza aprendizaje. Es importante que la didáctica de lenguas extranjeras se apoye en las TIC para ofrecer a los estudiantes los conocimientos y medios necesarios para desenvolverse competentemente en un mundo cada vez más globalizado. La multimedia ha permitido la aparición de prácticas pedagógicas novedosas, significativas e interconectadas que promueven la construcción de conocimiento a través de interacciones sincrónicas y asincrónicas con individuos y organizaciones. Esto conlleva la necesidad de un diseño tecno-pedagógico adecuado a las expectativas y necesidades de los estudiantes y que permita el desarrollo de sus capacidades lingüísticas y tecnológicas.

\section{Diseño instruccional y enseñanza de lenguas extranjeras}

Cada vez es más frecuente escuchar hablar de modelos instruccionales o tecno-pedagógicos. Existe una gran variedad de definiciones sobre lo que es el diseño instruccional (DI). Quizás una de las más amplias es la propuesta por Richey, Fields y Foson (2001, citado en Belloch, 2013) quienes afirman que el DI requiere una planificación instruccional sistemática que tenga en consideración aspectos tales como las necesidades, el desarrollo, la evaluación, la implementación y el mantenimiento de materiales y programas. En cualquier área del conocimiento, incluyendo la didáctica de lenguas extranjeras, es fundamental escoger el modelo de DI que mejor se ajuste a los objetivos educativos esperados. Es, por lo tanto, indispensable diseñar intervenciones pedagógicas, basadas en el contexto y perfil del aprendiz, que definan claramente los métodos y recursos implementados en cada fase planeada. En entornos de aprendizaje que incluyan el uso de herramientas tecnológicas, ya sea en modalidad e-learning, blended learning o mobile learning, será necesario tener en cuenta las potencialidades y limitaciones que este tipo de educación ofrece. Según Williams, Schrum, Sangrá 
y Guárdia (2017), para garantizar el éxito de los cursos diseñados y, por ende, la consecución de los objetivos de aprendizaje de los estudiantes, es necesario que su diseño instruccional ofrezca un alto grado de interactividad y calidad. En general, los modelos de diseño instruccional parten del análisis del contexto y del perfil del aprendiz para definir objetivos y procesos y, posteriormente, diseñar y seleccionar las actividades, los recursos y los contenidos idóneos con el fin de ofrecer una formación eficaz (Belloch, 2013). Existe una gran variedad de modelos pero para los propósitos del presente documento, se analizará exclusivamente el modelo ASSURE.

\section{$8 \quad$ Modelo de diseño instruccional ASSURE}

Este modelo de diseño instruccional, desarrollado por Heinich, Molenda, Russell y Smaldino (Williams et al. 2017), se fundamenta en la teoría constructivista y hace hincapié en el papel activo del estudiante. Propone actividades pedagógicas que permiten el aprendizaje en forma creativa y significativa (Belloch, 2013). Según la autora, las premisas constructivistas en que se basa este modelo son las siguientes (Figura 3):

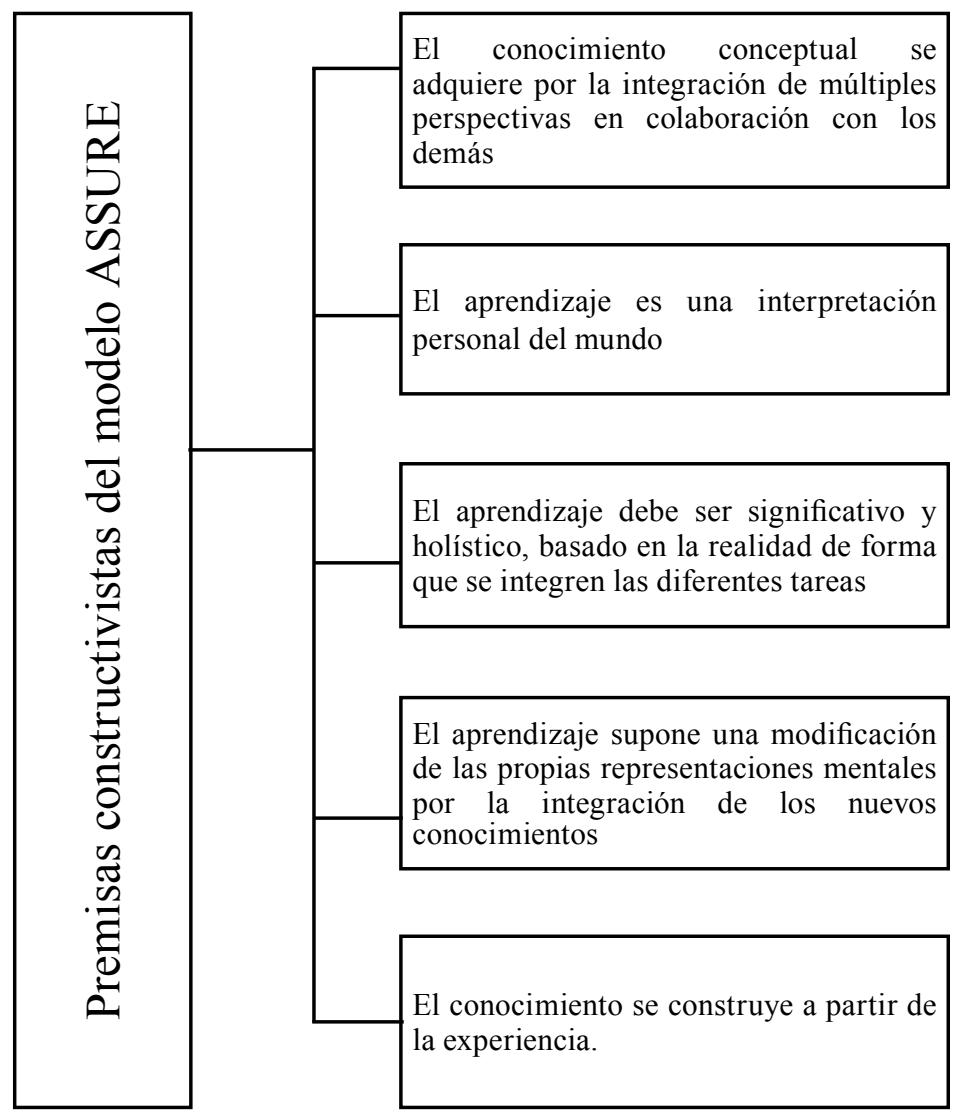

Figura 3. Premisas constructivistas del modelo ASSURE

Lo anterior implica que el diseño instruccional de una intervención pedagógica basada en el modelo ASSURE dará especial importancia a los conocimientos previos y motivaciones del estudiante; proveerá información relevante, coherente y significativa que permita la construcción de redes de 
significado y de entornos de aprendizaje motivadores que potencien el desarrollo de las competencias propuestas. Todo esto es especialmente significativo en el ámbito de la enseñanza y aprendizaje de lenguas extranjeras en donde es crucial la participación activa y propositiva del estudiante.

a Pasos o Fases del Modelo ASSURE: Los siguientes son los pasos o etapas del modelo ASSURE descritos por Williams et al. (2017, p. 50-51) a partir de la propuesta de Heinich, Molenda, Russel y Smaldino:

1) Analizar a los estudiantes (Analize), sus características generales, el conocimiento, las habilidades y las experiencias de las que parten y algo sobre sus estilos de aprendizaje. 2) Fijar objetivos (State), los resultados esperados del aprendizaje del curso y de cada unidad ya sea a nivel actitudinal, cognitivo o psicomotriz. 3) Seleccionar los métodos de formación, los documentos multimedia y los materiales (Select) que se van a utilizar, entre los que se pueden encontrar textos, imágenes fijas, vídeo, audio y material informático multimedia. 4) Utilizar los medios y materiales (Utilize) para posibilitar los objetivos que crearán los resultados deseados. 5) Exigir la participación de los alumnos (Require) para que la actividad no sea pasiva. Este es, sin lugar a dudas, uno de los pasos más relevantes para un docente de lengua extranjera ya que lo reta a buscar, seleccionar y diseñar actividades y recursos físicos y digitales que no sólo permitan sino que promuevan la participación activa, tanto a nivel oral como escrito, de sus estudiantes. Entre las actividades se pueden mencionar los debates, ejercicios en línea con autocorrección, creaciones colectivas y colaborativas, visualización de diferentes tipos de vídeos y tutoriales, entre otros. En cuanto a las herramientas TIC, es altamente recomendable el animar a los estudiantes a crear sus propios ambientes personales de aprendizaje (PLE, por su sigla en inglés) tal como lo propone Dávila (2015) para la enseñanza del español como lengua extranjera. En dichos PLE, los estudiantes se familiarizan con el uso de herramientas digitales para buscar y seleccionar información, crear conocimiento, publicarlo y compartirlo. 6) Evaluar y revisar (Evaluate) los aspectos formativos y sumativos de los alumnos y del formador.

\section{$9 \quad$ El modelo ASSURE y las TIC}

Según Dávila y Francisco (2007), el modelo instruccional ASSURE posibilita la planificación e implementación de instrucción apoyada en las TIC debido a que se enfoca en la planeación del entorno en el que se van a dar las actividades didácticas. Gustafson y Branch (2002, citado en Dávila y Francisco, 2007), argumentan que, aunque sus pasos se enfocan en los procesos educativos propios de entornos presenciales, se puede adaptar fácilmente a entornos educativos en línea sobre todo al momento de crear materiales y situaciones que estimulen la interactividad. Teniendo en cuenta que el estudiante puede interactuar con el contenido, con el docente y con sus pares (Williams et al. 2017), el diseñador instruccional de un curso de lengua extranjera debe proponer actividades como debates para que los estudiantes puedan expresar sus opiniones, interactuar con otros, evaluar los recursos ofrecidos y demostrar la conceptualización alcanzada. En otras palabras, deben diseñarse actividades y materiales que promuevan la interacción y la búsqueda y producción activa de información. Se deben proporcionar diversos medios tecnológicos para promover y facilitar este tipo de interacción significativa, colaborativa y motivadora.

Todo lo anterior, claramente permite la puesta en práctica de las recomendaciones pedagógicas para la enseñanza de una segunda lengua anteriormente descritas. Para permitir una transición exitosa del ámbito teórico a la práctica, Spencer (2015) sugiere las siguientes estrategias didácticas que, si bien es cierto se enfocan en la modalidad blended learning, pueden también adaptarse a modalidades de enseñanza e-learning y mobile learning: 
1) Adaptar los objetivos generales y estándares establecidos por las instituciones teniendo en cuenta la información arrojada por las encuestas anteriormente mencionadas. Esto permitirá definir los conocimientos y comportamientos esperados al finalizar las acciones formativas diseñadas. 2) Escoger TIC que estén alineadas en forma clara y coherente con los objetivos propuestos. El uso de la tecnología en el salón de clases debe responder a verdaderas necesidades de aprendizaje y no sólo al cumplimiento de requerimientos institucionales. Es crucial tener en cuenta las TIC que los estudiantes ya conocen y usan, o aquellas que por su naturaleza intuitiva sean de fácil manipulación tanto a nivel de trabajo individual como colaborativo. 3) Para asegurar una experiencia educativa provechosa, es indispensable realizar pruebas a las TIC que se van a usar en el contexto de clase y a las actividades diseñadas con base en dichos recursos digitales. Esto facilitará su posterior uso en las actividades planeadas y permitirá a los estudiantes desarrollar mayor seguridad y motivación al usarlas. 4) Involucrar a los estudiantes en actividades que les permitan tener un papel protagónico y activo en cada una de las acciones pedagógicas implementadas, sobre todo aquellas de tipo virtual. La creación de identidades y comunidades virtuales permitirá un apoyo efectivo entre pares sin importar su ubicación en tiempo real. El docente debe estar solicitando constantemente la retroalimentación de los estudiantes en cuanto a las herramientas tecnológicas y las tareas desarrolladas con estos recursos para determinar su eficacia y realizar los ajustes que sean necesarios. 5)Comprender y aceptar que la implementación del modelo instruccional ASSURE será siempre una obra en progreso que seguramente deberá irse adaptando a las necesidades cambiantes de los estudiantes y a los avances tecnológicos de los dispositivos, apps y software incorporados en el diseño inicial del programa educativo. Igualmente, es importante realizar las modificaciones del caso a las actividades evaluativas presenciales y virtuales, los contenidos diseñados o seleccionados y el grado de integración de las herramientas tecnológicas en las actividades de clase.

\section{Consideraciones finales y conclusiones}

El modelo de diseño instruccional ASSURE permite la planeación de actividades pedagógicas congruentes con los estándares de enseñanza de una segunda lengua propuestos por el MEN, ya que no sólo facilita sino que exige la participación activa del estudiante en las diferentes etapas de su proceso de aprendizaje y el uso efectivo de las TIC para alcanzar los objetivos educativos propuestos.

Como se describió anteriormente, desde la perspectiva constructivista, es deseable que el estudiante de lengua extranjera pueda construir las redes de conocimiento a partir de interacciones comunicativas significativas e interesantes que le lleven a asumir un papel protagónico en sus procesos de aprendizaje. Por consiguiente, la labor del diseñador instruccional, generalmente el propio docente, será determinante ya que de la apropiada selección de materiales auténticos y de actividades motivantes y relevantes dependerá en parte el éxito de las iniciativas de corte constructivista y comunicativo que por medio de este modelo se propongan. La autenticidad de los materiales, la calidad y grado de realidad y relevancia de las interacciones propuestas permitirán la construcción de redes de conocimiento en la segunda lengua que facilitarán una comunicación lingüística, social y culturalmente adecuada.

Aunque las bondades de este modelo de diseño instruccional en el área de la enseñanza de idiomas son evidentes, es claro que se requiere el diseño e implementación de diversos programas de enseñanza de una segunda lengua en diferentes entornos y en diferentes modalidades para refinar aún más su eficacia. Esto debido a que, por ejemplo, el grado de interacción personal que el estudiante tenga con su docente generalmente incide en la consecución de los logros propuestos. En ese orden de ideas, sería recomendable profundizar en el grado de eficiencia que el modelo ASSURE tiene en las modalidades e-learning y blended learning en la enseñanza de lenguas extranjeras para determinar 
hasta qué punto es deseable o no la planeación de actividades presenciales y qué tan efectivo puede ser el apoyo brindado por las TIC a las mismas.

El dominar una segunda lengua es, hoy en día, una necesidad académica y profesional para millones de personas en todo el mundo que necesitan acceder a conocimientos científicos, culturales y tecnológicos disponibles en otro idioma. Un adecuado diseño instruccional, sobre todo uno que tenga en cuenta la participación activa del estudiante y el uso eficiente de las TIC, como lo hace el modelo ASSURE, permitirá la instrumentalización de una pedagogía de las lenguas extranjeras cada vez más innovadora y enfocada en las necesidades de formación de los estudiantes.

\section{Bibliografía}

Altablero (2005). Para vivir en un mundo global. Ministerio de Educación Nacional. Recuperado de http://www.mineducacion.gov.co/1621/article-97500.html

Belloch, C. (2013). Modelos de diseño instruccional. Universitat de Valencia. Recuperado de https:// www.uv.es/bellochc/pedagogia/EVA4.wiki?1

Dávila, A. y Francisco, J. (2007). Diseño instruccional de la educación en línea usando el Modelo ASSURE. EDUCARE, 11, 3. UPEL, Barquisimeto, Venezuela. Recuperado de http://revistas. upel.edu.ve/index.php/educare/article/view/22

Dávila, A., Ruiz B., C. y Francisco, J. (2014). Modelo tecno-pedagógico para la implantación de la modalidad semipresencial en la educación universitaria. EDUCARE, 17,3. Barquisimeto, Venezuela. Recuperado de http://revistas.upel.edu.ve/index.php/educare/article/view/1171

Dávila, C. (2015) Comenzar a crear el entorno personal de aprendizaje en la clase de español. Instituto Cervantes, Nápoles. Recuperado de http://cvc.cervantes.es/ensenanza/biblioteca_ele/ publicaciones_centros/PDF/napoles_2015/02_davil a.pdf

Ecured. Aprendizaje. Recuperado el 25/05/2017, de https://www.ecured.cu/Aprendizaje

Ministerio de Educación Cultura y Deporte de España. (NA de Junio de 2002). Marco Común Europeo de Referencia para las Lenguas: Aprendizaje,Enseñanza, Evaluación. (S. G. Internacional, Ed.). Recuperado de http://www.mineducacion.gov.co/1621/articles237704_archivo_pdf_marco_ europeo.pdf

MEN. Ministerio de Educación Nacional. (s.f.). Idiomas Extranjeros. Serie Lineamientos Curriculares. Bogotá (Col). Recuperado el 29 de junio de 2017, de: https://www.mineducacion.gov.co/1759/ articles-339975 recurso 7.pdf

Spencer, R. (2015) 6 Tips To Apply The ASSURE Model In Blended Learning. Elearning Industry. Obtenido de https://elearningindustry.com/6-tips-apply-assure-model-blended-learning

Trujillo, F. (2001). Objetivos en la enseñanza de lenguas extranjeras: De la competencia lingüística a la competencia intercultural. Facultad de Educación y Humanidades de Ceuta. Universidad de Granada. Recuperado de http://www.ugr.es/ ftsaez/aspectos/objetivos.pdf

Williams, P., Schrum, L., Sangrà, A., \& Guàrdia, L. Modelos de diseño instrucciona (módulo didáctico UOC-lectura del módulo 2). Recuperado de http://materials.cv.uoc.edu/cdocent/ WSKAIKJT5EW8JJ40YA4B.pdf 